\title{
THE MEASUREMENT OF Q' AND Q" IN THE CERN-SPS BY HEAD-TAIL PHASE SHIFT ANALYSIS
}

\author{
R. Jones, H. Schmickler, CERN, Geneva, Switzerland
}

\begin{abstract}
A so-called "Head-Tail" chromaticity measurement system has recently been installed in the CERN-SPS, which allows the chromaticity $\left(\mathrm{Q}^{\prime}\right)$ to be calculated from several hundred turns of data after transverse excitation. The measurement relies on the periodic dephasing and rephasing that occurs between the head and tail of a single bunch for non-zero chromaticity. By measuring the turnby-turn position data from two longitudinal positions in a bunch it is possible to extract the relative dephasing of the head and the tail, and so to determine the chromaticity. In addition, by changing the orbit of the circulating beam this technique allows the variation of chromaticity with radial position $\left(\mathrm{Q}^{\prime \prime}\right)$ to be measured with a much higher resolution than is currently possible using RF modulation. This paper describes this "Head-Tail" measurement technique and discusses some recent results obtained using prototype LHC beam (25ns spacing) in the CERNSPS.
\end{abstract}

\section{INTRODUCTION}

The tight tolerances on beam parameters required for successful LHC operation implies a good knowledge of the chromaticity throughout the cycle. However, many of the methods currently used to measure chromaticity in circular machines (see [1] and references therein) are likely to be incompatible with LHC high intensity running. For example, the most common method, of measuring the betatron tune as a function of beam energy, might be difficult to implement due to the tight tolerances imposed on the betatron tune itself and the limited momentum acceptance of the LHC. Chromaticity can also be calculated from the amplitude of the synchrotron sidebands observed in the transverse frequency spectrum. This method, however, suffers from resonant behaviour not linked to chromaticity and the fact that the low synchrotron tune of the LHC would make it difficult to distinguish these side-bands from the main betatron tune peak. The width of the betatron tune peak itself, or the phase response of the beam transfer function also give a measure of chromaticity, but require a knowledge of how the momentum spread in the beam changes with energy.

In this paper we describe the first results from an alternative method, tested during 2000 on the CERN-SPS. This so-called "Head-Tail" chromaticity technique does not rely on an accurate knowledge of the fractional part of the betatron tune and, for a machine operating well above transition, the calculated chromaticity is virtually independent of beam energy.

\section{THE HEAD-TAIL PRINCIPLE}

Assuming longitudinal stability, a single particle will rotate in longitudinal phase-space at a frequency equal to the synchrotron frequency. During this longitudinal motion the particle also undergoes transverse motion, which can be described by the change in the betatron phase, $\theta(\mathrm{t})$, along the synchrotron orbit. If the whole bunch is kicked transversely, then the resulting transverse oscillations for a given longitudinal position within the bunch can be shown to be given by

$$
\mathrm{y}(\mathrm{n})=\mathrm{A} \cos \left[2 \pi \mathrm{nQ} \mathrm{Q}_{0}+\omega_{\xi} \hat{\tau}\left(\cos \left(2 \pi \mathrm{nQ}_{\mathrm{S}}\right)-1\right)\right]
$$

where $\mathrm{n}$ is the number of turns since the kick, $\mathrm{Q}_{0}$ is the betatron tune, $Q_{s}$ is the synchrotron tune, $\hat{\tau}$ is the longitudinal position with respect to the centre of the bunch, and $\omega_{\xi}$ is the chromatic frequency and is given by

$$
\omega_{\xi}=Q^{\prime} \omega_{0} \frac{1}{\eta}
$$

Here Q' is the chromaticity, $\omega_{0}$ is the revolution frequency and $\eta=1 / \gamma^{2}-1 / \gamma_{\text {tr }}^{2}$. If we now consider the evolution of two longitudinal positions within a single bunch separated in time by $\Delta \tau$, then from Eq. 1 it follows that the phase difference in the transverse oscillation of these two positions is given by

$$
\Delta \Psi(\mathrm{n})=-\omega_{\xi} \Delta \tau\left(\cos \left(2 \pi \mathrm{nQ}_{\mathrm{S}}\right)-1\right)
$$

This phase difference is a maximum when $\mathrm{nQ}_{S}=1 / 2$, i.e. after half a synchrotron period, giving

$$
\Delta \Psi_{\mathrm{MAX}}=-2 \omega_{\xi} \Delta \tau
$$

The chromaticity can therefore be written as

$$
\begin{gathered}
\mathrm{Q}^{\prime}=\frac{-\eta \Delta \psi(\mathrm{n})}{\omega_{0} \Delta \tau\left(\cos \left(2 \pi \mathrm{nQ}_{\mathrm{s}}\right)-1\right)} \\
\mathrm{Q}^{\prime}=\frac{\eta \Delta \psi_{\mathrm{MAX}}}{2 \omega_{0} \Delta \tau}
\end{gathered}
$$

\section{THE SPS HEAD-TAIL MONITOR}

A schematic layout of the SPS Head-Tail monitor setup is shown in Fig. 1. A straight stripline coupler followed by a $180^{\circ}$ hybrid is used to provide the sum and 
difference signals for a given measurement plane. These signals are fed into a fast-sampling (2GS/s on each channel), high bandwidth $(2 \mathrm{GHz})$ digital oscilloscope. A VME front-end acquisition crate then retrieves the data via a GPIB link. All the oscilloscope and acquisition parameters are accessible from the SPS control room through a UNIX graphical interface.

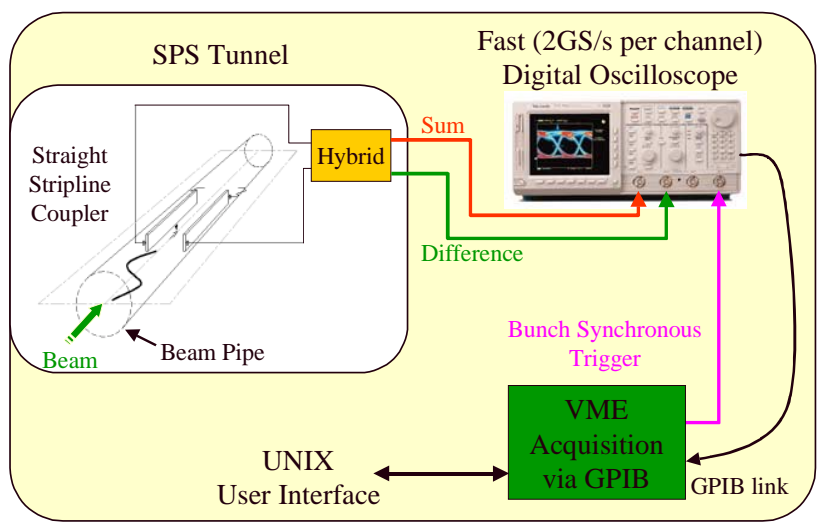

Figure 1: Layout of the SPS Head-Tail Monitor

The oscilloscope is triggered using bunch synchronous timing. Using the "Fast-Frame" capabilities of the oscilloscope allows $25 \mathrm{~ns}$ of data (corresponding to the LHC bunch spacing) to be captured on each SPS revolution. In this way the evolution of the signal from a single, specific bunch can be tracked over 372 turns (the memory limit of the oscilloscope). Since the bunch synchronous timing has a peak-peak jitter of $12 \mathrm{~ns}$, the sum signal is used to re-align the difference signal for each turn. In this way, the timing jitter is reduced to well below the sampling frequency.

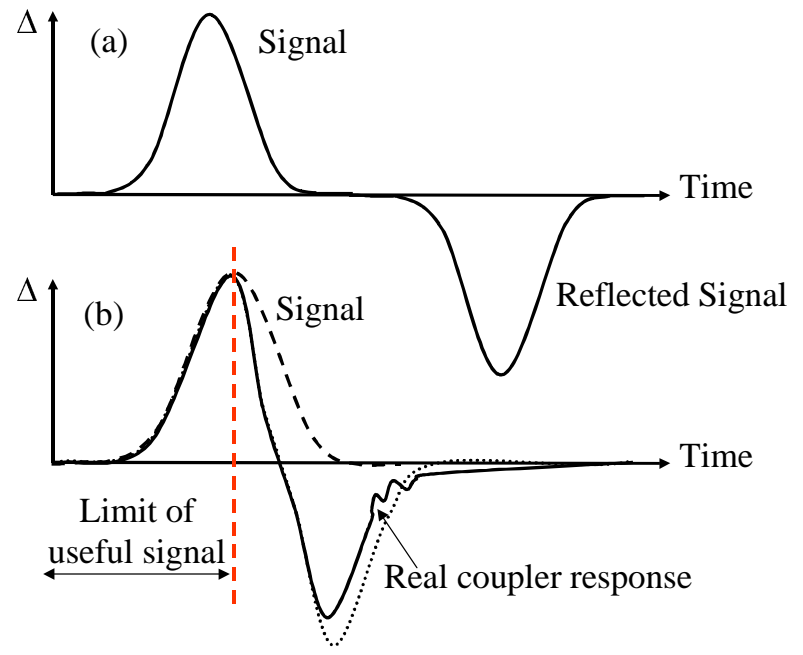

Figure 2: Typical coupler response.

a) total bunch length $<2 \times$ coupler length.

b) total bunch length $>2 \times$ coupler length.

Fig. 2 shows some typical signals obtained from the coupler. If the total bunch length is less than twice the coupler stripline length, Fig 2(a), then the signal from the bunch and its reflection from the opposite end of the stripline are well separated in time. If, however, the bunch is longer than twice the stripline length then the signal and reflection are no longer separated, Fig. 2(b), and the useful part of the signal is limited. This latter case was true for the SPS set-up during 2000, where the typical bunch length was $\sim 4 \mathrm{~ns}$ and twice the coupler length corresponded to $2.5 \mathrm{~ns}$. Hence measurements on the tail of the bunch were not possible, and therefore all the results shown are for measurements of the head and centre of the bunch.

All measurements were performed using an LHC batch of 84 bunches with $25 \mathrm{~ns}$ bunch spacing, a bunch intensity of $\sim 2 \times 10^{10}$ protons, accelerated from $26 \mathrm{GeV}$ to $450 \mathrm{GeV}$. Each measurement was performed on-line on one bunch after the application of a single transverse kick using an SPS Q-kicker.

\section{RESULTS}

An example of a Head-Tail monitor acquisition is shown in Fig 3. Fig. 3(a) and 3(b) show the transverse evolution of the head and tail (or more precisely the head and centre) of the bunch after the application of a single kick. By performing phase demodulation using a Hilbert transformation on this data, the relative phase difference (Fig. 3(c)) of the head and tail of the bunch can be extracted. The periodic phasing and dephasing between head and tail, at a frequency corresponding to the synchrotron tune, is clearly visible. In this case it is only the head that changes its relative phase, since the tail corresponds to the centre of the bunch, which does not undergo a dephasing with respect to the betatron tune. Using the head-tail phase difference and Eq. 5 it is possible to calculate the chromaticity. The result is shown in Fig. 3(d). Due to cosine term in the denominator of Eq. 5 , the error in the calculation is large around multiples of the synchrotron tune. Hence only the points around the maximum of the dephasing are used to obtain the final, average value for the chromaticity.

The usual way in which to measure chromaticity in the

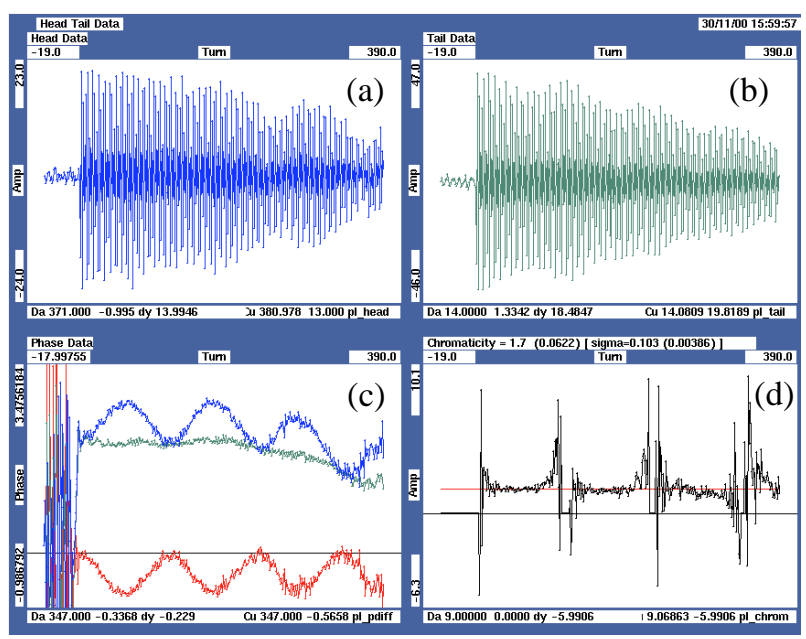

Figure 3: CERN-SPS Head-Tail monitor acquisition.

a) head oscillations; b) tail oscillations;

c) head-tail phase difference; d) chromaticity 
SPS is by changing the RF frequency, and hence the beam momentum, and measuring the corresponding change in the betatron tune. In practice, this implies three measurements performed for three different RF settings, taking place over several SPS supercycles. Due to the momentum change imposed by each RF setting, the beam has different transverse positions, hence the term "radial steering" chomaticity measurement.

Fig. 4 shows the results obtained from this traditional technique compared to those using the head-tail method, as a function of chromaticity. For each chromaticity setting, several measurements were performed using the radial steering technique, each implying three different RF frequency settings corresponding to three different transverse positions. The head-tail measurement was acquired for all RF settings.

A very good linearity is seen to exist between the headtail and radial steering techniques when the measurement is performed for the same transverse beam position. However, the absolute value of the chromaticity measured via the head-tail technique is approximately a factor of two smaller than would be expected. Measurements carried out for several different energies between $26 \mathrm{Gev}$ and $400 \mathrm{GeV}$ all showed this good linearity, but required correction factors ranging from 1.6 to 2.4. No firm explanation can be put forward at the moment to explain this missing factor, however this could be caused by bandwidth limitations in the acquisition electronics or the fact that the theoretical model relies on single particle dynamics, and therefore does not take into account any collective effects.

Another thing that can be remarked from Fig. 4 is the

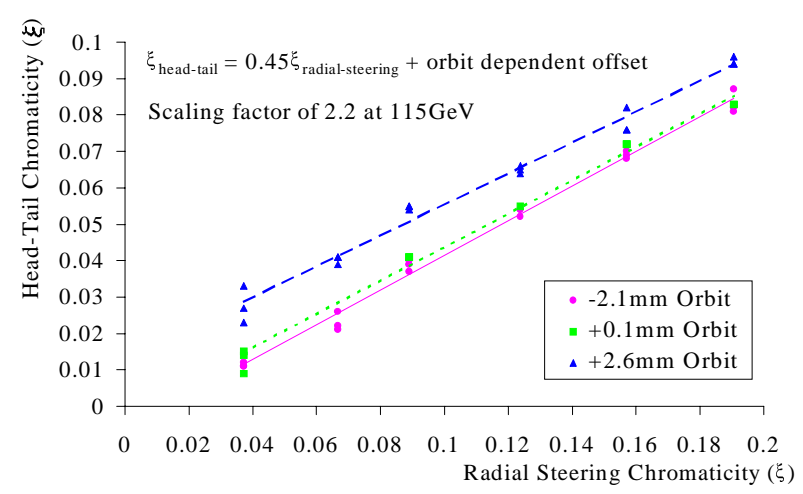

Figure 4: Comparison of chromaticity measured by radial steering and the head-tail technique.

fact that the head-tail measurement is able to distinguish the chromaticity for different radial positions of the beam. This makes it possible to use this technique measure the variation of chromaticity with position, or Q', of the machine. The results from such a measurement is shown in Fig. 5. Here the RF frequency was changed in much smaller steps than for a traditional chromaticity measurement, so as to build up a picture of the Q" of the SPS. The results from radial steering measurements are included for completeness, but have very large error bars due to the nearly immeasurable changes in betatron tune induced by such small RF changes. The head-tail measurements were scaled by the appropriate factor to allow a comparison with the radial steering, and can be seen to give a very accurate picture of the variation of chromaticity with radial position.

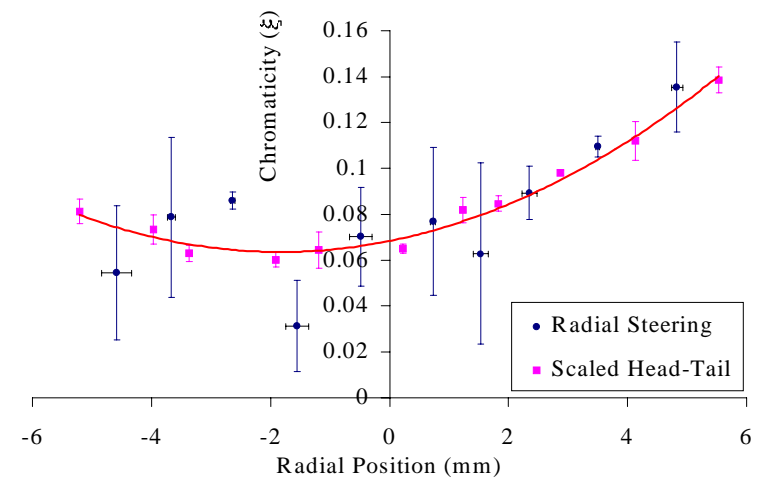

Figure 5: The measurement of second order chromaticity (Q') using head-tail phase shift analysis.

A further advantage of the head-tail technique is that the whole measurement can be performed during a single synchrotron period, which in the SPS corresponds to between 100-300 turns. This means that it is possible to measure the chromaticity throughout the cycle, with the only limitation being the emittance blow-up induced by each applied kick. Measurement at $0.5 \mathrm{~Hz}$ has been achieved at the SPS, a rate which is determined solely by the GPIB data transfer rate and re-arm time of the oscilloscope.

\section{CONCLUSIONS}

It has been demonstrated that the chromaticity of a proton synchrotron can be measured in an operational way from the evolution of the phase difference between the head and tail of a single bunch after the application of a transverse kick. In addition this head-tail technique provides an accurate method for investigating the second order chromaticity of such a machine. Further studies are planned to pursue the origins of the scaling factor, the effects of higher order fields, transverse coupling, and also to determine the signal to noise ratio required for accurate measurements.

\section{REFERENCES}

[1] Schmickler, H., "Diagnostics and Control of the Time Evolution of Beam Parameters" (CERN-SL-97-68), presented at the 3rd European Workshop on Beam diagnostics and Instrumentation for Particle Accelerators (DIPAC 97), Frascati, Italy, October 1997.

[2] D. Cocq, O. R. Jones, H. Schmickler, "The Measurement of Chromaticity via a Head-Tail Phase Shift", 8th Beam Instrumentation Workshop BIW '98, Stanford, CA, USA, May 1998. 